Guillermo Reyes · Juan Luis Vázquez

\title{
A weighted symmetrization for nonlinear elliptic and parabolic equations in inhomogeneous media
}

Received September 20, 2005 and in revised form December 21, 2005

\begin{abstract}
In the theory of elliptic equations, the technique of Schwarz symmetrization is one of the tools used to obtain a priori bounds for classical and weak solutions in terms of general information on the data. A basic result says that, in the absence of lower-order terms, the symmetric rearrangement of the solution $u$ of an elliptic equation, that we write $u^{*}$, can be compared pointwise with the solution of the symmetrized problem. The main question we address here is the modification of the method to take into account degenerate equations posed in inhomogeneous media. Moreover, the equations we want to deal with involve weights that make them of non-divergence form, at least when written in terms of the natural variables. We find comparison results covering the elliptic case and the corresponding evolution models of parabolic type, with emphasis on equations of porous medium type. More specifically, we obtain a priori bounds and decay estimates for wide classes of solutions of those equations.
\end{abstract}

Keywords. Nonlinear elliptic and parabolic equations, degenerate equations, inhomogeneous media, symmetrization, concentration comparison

\section{Introduction}

The technique of Schwarz symmetrization is a well known tool in the theory of elliptic equations that is used to obtain a priori bounds for classical and weak solutions in terms of general information on the data. A basic result of symmetrization theory says that, in the absence of lower-order terms, the symmetric rearrangement $u^{*}$ of the solution $u$ of a linear, uniformly elliptic equation with zero boundary data which can be compared pointwise with the solution of the rearranged (or symmetrized) problem. See Section 2 for the precise definitions.

Combining the classical result with the technique of concentration comparison, it is proved in the survey [V4] that, if a (possibly nonlinear) zero-order term is present, one can still get comparison in the sense of concentrations (hence, in the sense of any $L^{p}$ norm, $p \geq 1$, or any Orlicz norm in balls, and in the uniform norm) between the rearranged solution and the solution of the rearranged problem. For this to hold, some restrictions

G. Reyes: Departamento de Matemáticas, Universidad Carlos III de Madrid, 28911 Leganés, Spain; e-mail: greyes@math.uc3m.es

J. L. Vázquez: 1 Departamento de Matemáticas, Universidad Autónoma, 28049 Madrid, Spain; e-mail: juanluis.vazquez@uam.es

Mathematics Subject Classification (2000): 35J60, 35K55, 35K65, 35B05, 35B40, 47H20 
on the zero-order term are needed. Fortunately, such restrictions are met by the elliptic problems that arise when solving the evolution problems that interest us by means of a backwards-in-time difference scheme, i.e., the so-called Crandall-Liggett implicit scheme. In particular, a wide class of nonlinear, degenerate parabolic equations fall under the scope of the method, since a simple change of unknown allows hiding the nonlinearity in the elliptic zero-order term. As a result, one can obtain a priori estimates for the solutions to the Cauchy or Cauchy-Dirichlet problem with zero boundary data for these evolution equations.

The main question we address here is the modification of the above method to take into account two aspects of current interest in applications: the equations are degenerate elliptic or parabolic, and they are posed in inhomogeneous media. Moreover, the equations we want to deal with involve weights that make them of non-divergence form, at least when written in terms of the natural variables. We will find comparison results covering both issues, namely the elliptic equations

$$
-\frac{1}{\rho(x)} \sum_{i, j} \partial_{i}\left(a_{i j}(x) \partial_{j} u\right)=g(x, u)
$$

and the corresponding evolution models of parabolic type

$$
\rho(x) u_{t}=\sum_{i, j} \partial_{i}\left(a_{i j}(x) \partial_{j} \phi(u)\right)+g(x, t) .
$$

The latter equation has been proposed and studied in connection with filtration of fluids in inhomogeneous porous media or thermal propagation in plasma (cf. [KR1]). The main technical novelty in the proof is the use of symmetrization with respect to the measure with density $\rho(x)$, which we will assume "almost radial" in the sense that there exist a radially symmetric function $\rho_{0}$ and a constant $0<c \leq 1$ such that

$$
c \rho_{0} \leq \rho \leq \rho_{0}
$$

in the domain under consideration. The use of symmetrization techniques allows us to derive a priori estimates for the solutions of these problems in terms of the norms of the data. These estimates are the goal of the present work since they represent a main step in the construction of general weak theories.

The paper is organized as follows: Section 2 is devoted to the main definitions and facts that we shall be using throughout. Sections 3 and 4 deal with the elliptic problems: in Section 3 we obtain the pointwise estimate when no zero-order terms are present, while in Section 4 the proof is adapted so as to keep track of (a special class of) zero-order terms, and the concentration comparison result is proved. This ends the study of elliptic symmetrization.

The last part of the paper is devoted to the application of the preceding results to obtain comparison results and a priori estimates for parabolic problems. In Section 5 we combine the results of the preceding section and the semigroup approach and prove the corresponding concentration comparison result for parabolic problems. In Section 6 we apply this comparison result to the Cauchy problem for an inhomogeneous porous medium equation. A final appendix includes the proof of one important inequality used throughout. 


\section{Functional preliminaries}

This section collects the main general ideas that we shall be using. We start by a review of the standard definitions. Let $\Omega$ be a domain in $\mathbb{R}^{n}$, not necessarily bounded, possibly $\mathbb{R}^{n}$. $\mathcal{L}(\Omega)$ is the set of [classes of] Lebesgue measurable real functions defined in $\Omega$ up to a.e. equivalence. For every function $f \in \mathcal{L}(\Omega)$, we define the distribution function $\phi_{f}$ of $f$ by the formula

$$
\phi_{f}(k)=\operatorname{meas}\{x:|f(x)|>k\},
$$

where meas means the Lebesgue measure in $\mathbb{R}^{n}$ and $k>0$. We denote by $\mathcal{L}_{0}(\Omega)$ the space of measurable functions in $\Omega$ such that $\phi_{f}(k)$ is finite for every $k>0$. If $\Omega$ has finite measure, then $\mathcal{L}_{0}(\Omega)=\mathcal{L}(\Omega)$, otherwise $\mathcal{L}_{0}(\Omega)$ contains the measurable functions that tend to zero at infinity in a weak sense. All $L^{p}(\Omega)$ spaces with $1 \leq p<\infty$ are contained in $\mathcal{L}_{0}(\Omega)$.

\subsection{Rearrangement}

A measurable function $f$ defined in $\mathbb{R}^{n}$ is called radially symmetric (or radial for short) if $f(x)=\tilde{f}(r), r=|x|$. It is called rearranged if it is nonnegative, radially symmetric, and $\tilde{f}$ is a nonincreasing function of $r>0$. For definiteness, we also impose that $\tilde{f}$ be leftcontinuous at every jump point. We will often write $f(x)=f(r)$ by abuse of notation. A similar definition applies to functions defined on a ball $B=B_{R}(0)=\left\{x \in \mathbb{R}^{n}:|x|<R\right\}$.

\subsection{Symmetrization}

For every bounded domain $\Omega$ the symmetrized domain is the ball $\Omega^{*}=B_{R}(0)$ having the same volume as $\Omega$, i.e.,

$$
\operatorname{meas}(\Omega)=\omega_{n} R^{n}
$$

where $\omega_{n}$ is the volume of the unit ball in $\mathbb{R}^{n}$. For a function $f \in \mathcal{L}_{0}(\Omega)$ we define the spherical rearrangement of $f$ (also called the symmetrized function of $f$ ) as the unique rearranged function $f^{*}$ defined in $\Omega^{*}$ which has the same distribution function as $f$, i.e., for every $k>0$,

$$
\phi_{f}(k):=\operatorname{meas}\{x \in \Omega:|f(x)|>k\}=\operatorname{meas}\left\{x \in \Omega^{*}: f^{*}(x)>k\right\} .
$$

The quantity is finite for every $k>0$ by the assumption $f \in \mathcal{L}_{0}(\Omega)$. Then

$$
f^{*}(x)=\inf \left\{k>0: \operatorname{meas}\{y:|f(y)|>k\}<\omega_{n}|x|^{n}\right\} .
$$

A rearranged function coincides with its spherical rearrangement. Sometimes the name symmetric decreasing rearrangement is used. The following Hardy-Littlewood formula is well known and illustrates the relation between $f$ and $f^{*}$ :

$$
\int_{B_{R}(0)} f^{*} d x=\sup \left\{\int_{E}|f| d x: E \subset \Omega, \operatorname{meas}(E) \leq \operatorname{meas}\left(B_{R}\right)\right\} .
$$


There is also an immediate relation between distribution functions and $L^{p}$ integrals given by the formulas

$$
\int_{\Omega}|f|^{p} d x=-\int_{0}^{\infty} k^{p} d \phi(k)=p \int_{0}^{\infty} k^{p-1} \phi(k) d k
$$

and

$$
\int_{\Omega \cap\{|f| \geq a\}}|f|^{p} d x=-\int_{a}^{\infty} k^{p} d \phi(k)=p \int_{a}^{\infty} k^{p-1} \phi(k) d k+a^{p} \phi(a) .
$$

Since the distribution functions of $f$ and $f^{*}$ are identical, conservation of integrals $\int_{\Omega}|f|^{p} d x=\int_{\Omega^{*}}\left(f^{*}\right)^{p} d x$ holds for every $p \in[1, \infty)$. Moreover, for every convex, nonnegative and symmetrical real function $\Phi$,

$$
\int_{\Omega} \Phi(f) d x=\int_{\Omega^{*}} \Phi\left(f^{*}\right) d x
$$

Note that $f^{*}$ is continuous if $f$ is.

\subsection{Symmetrization with respect to a measure}

As a natural generalization of the standard notions, one can introduce symmetrization with respect to a measure $\mu$ defined on the domain $\Omega$. As the symmetrized domain $\Omega_{\mu}^{*}$ we take the ball $B_{R}(0)$ with Lebesgue measure equal to the $\mu$-measure of $\Omega$ :

$$
\operatorname{meas}\left(\Omega_{\mu}^{*}\right)=\mu(\Omega)
$$

(so that $\Omega_{\mu}^{*}=\mathbb{R}^{n}$ if $\mu(\Omega)=\infty$ ). Formulas 2.1 and 2.3 should be replaced by

$$
\phi_{f ; \mu}(k):=\mu\{x \in \Omega:|f(x)|>k\}=\operatorname{meas}\left\{y \in \Omega_{\mu}^{*}: f_{\mu}^{*}(y)>k\right\} .
$$

Observe that we have kept the usual Lebesgue measure on the right-hand side. If $\mu$ is not the Lebesgue measure, a rearranged function, in the sense defined above, will not coincide with its $\mu$-rearrangement, since an extra contraction/dilation will take place with respect to the space variable. The equality of Orlicz norms 2.8 holds in the form

$$
\int_{\Omega} \Phi(f) d \mu=\int_{\Omega_{\mu}^{*}} \Phi\left(f_{\mu}^{*}\right) d y .
$$

The weighted version of the Hardy-Littlewood inequality reads

$$
\int_{B_{R}(0)} f_{\mu}^{*} d y=\sup \left\{\int_{E}|f| d \mu: E \subset \Omega, \mu(E) \leq \operatorname{meas}\left(B_{R}\right)\right\} .
$$

A proof of this formula under very general assumptions including as a particular case weighted symmetrization can be found in [CRZ]. For the reader's convenience, we have devoted a final appendix to a proof of this important inequality in our case. 


\subsection{Mass concentration}

The comparison of mass concentrations is a basic notion in our approach to getting estimates for elliptic and parabolic equations. The precise definition that was introduced in [V1] is as follows:

Definition 2.1. Given two radially symmetric functions $f, g \in L_{\mathrm{loc}}^{1}\left(\mathbb{R}^{n}\right)$ we say that $f$ is more concentrated than $g, f \succ g$, if for every $R>0$,

$$
\int_{B_{R}(0)} f(x) d x \geq \int_{B_{R}(0)} g(x) d x,
$$

i.e.,

$$
\int_{0}^{R} f(r) r^{n-1} d r \geq \int_{0}^{R} g(r) r^{n-1} d r .
$$

The partial order relationship $\succ$ will be called comparison of mass concentrations. We can also write $f \succ g$ in the form $g \prec f$. A similar definition applies to radially symmetric and locally integrable functions defined on a ball $B=B_{R}(0)$. In the case of rearranged functions this notion coincides with the comparison introduced by Hardy and Littlewood [HLP], which is also used by Bandle in her book [Ba2]; but the present definition does not require the functions to be rearranged, only radially symmetric, and the difference is used below. In fact, the natural way of looking at the concept is to view it as a comparison between two radially symmetric measures, $d \mu_{f}=f(x) d x$ and $d \mu_{g}=g(x) d x$. Then the comparison reads

$$
\mu_{f}\left(B_{R}(0)\right) \geq \mu_{g}\left(B_{R}(0)\right) \quad \text { for every } R>0 .
$$

In this formulation, comparison can be considered for general radially symmetric Radon measures. Measures are natural data for elliptic and parabolic equations. The comparison of concentrations can be formulated in an equivalent way when the functions are rearranged, thanks to a powerful equivalence result, which seems to be essentially due to Hardy and Littlewood. The precise formulation is the following.

Lemma 2.2. Let $f, g \in L^{1}(\Omega)$ be rearranged functions defined in $\Omega=B_{R}(0)$ and assume $g \rightarrow 0$ as $|x| \rightarrow R$. Then $f \succ g$ if and only if for every convex nondecreasing function $\Phi:[0, \infty) \rightarrow[0, \infty)$ with $\Phi(0)=0$ we have

$$
\int_{\Omega} \Phi(f(x)) d x \geq \int_{\Omega} \Phi(g(x)) d x .
$$

The result is also valid when $R=\infty$ and $f, g \in L_{\mathrm{loc}}^{1}\left(\mathbb{R}^{n}\right), g \rightarrow 0$ as $|x| \rightarrow \infty$.

We will make use of the following two results, which are adaptations to our case of Proposition 4.1 (respectively Theorem 4.6) in [V4].

Theorem 2.3. Let $f(r)$ be a radially symmetric and decreasing function in $L_{\mathrm{loc}}^{1}(\Omega)$ and let $u(r)$ be a solution of the equation

$$
-\Delta u+\beta(u)=f,
$$


where $\beta$ is an increasing function in $[0, \infty)$ with $\beta(0)=0$. Let $v=\beta(u)$. There is an alternative:

(i) Standard case: $f \succ v$, and then $v$ and $u$ are nonincreasing;

(ii) Increasing case: $f \succ v$ does not hold, and then $u$ is increasing in $r$ in an interval $I=(a, R), v$ is nondecreasing with $v>f$ in $I$, and

$$
\int_{\Omega}(v(x)-f(x)) d x>0 .
$$

Moreover, in this case the function $X(r):=\int_{0}^{r} s^{n-1}(v(s)-f(s)) d s$ is positive and increasing in $I$.

The next theorem makes use of the concepts of integral sub- and supersolutions: a radial integral subsolution of equation 2.16 is a radial function $u \in W_{\mathrm{loc}}^{1,1}(\Omega)$ with $\beta(u) \in$ $L_{\mathrm{loc}}^{1}(\Omega)$ and such that

$$
f \succ-\Delta u+\beta(u) .
$$

Much in the same way we define a radial integral supersolution, by reversing the concentration order in (2.18). See more details in [V4, Section 4].

Theorem 2.4. Let $f_{i}(r), i=1,2$, be two radial functions in $L_{\mathrm{loc}}^{1}(\Omega)$, let $\beta$ be an increasing function in $[0, \infty)$ with $\beta(0)=0$ and let $u_{1}(r)$ be an integral supersolution and $u_{2}(r)$ an integral subsolution of the equation

$$
-\Delta u_{i}+\beta\left(u_{i}\right)=f_{i},
$$

Put $v_{i}=\beta\left(u_{i}\right)$. If $f_{1} \succ f_{2}$, and either $v_{1}$ or $v_{2}$ is nondecreasing, then either (i) $v_{1} \succ v_{2}$, or (ii) the quantity

$$
V(r)=\int_{0}^{r}\left(v_{2}(s)-v_{1}(s)\right) s^{n-1} d s
$$

is positive and increasing for all large $r, v_{2}(r)-v_{1}(r)$ is nonnegative and $u_{2}-u_{1}$ is strictly increasing.

Roughly speaking, the first result says that, in the standard case, the solution is less concentrated than the data, while the second result says that to more concentrated data correspond more concentrated solutions.

\section{Symmetrization and comparison. Elliptic problems I}

The classical result of symmetrization theory deals with uniformly elliptic equations in a bounded domain with Dirichlet data, or in the whole space; it says that the symmetric rearrangement $u^{*}$ of a solution $u$ can be compared pointwise with the solution of a certain symmetrized problem involving the Laplacian operator and the symmetrized function of $f$. We present here the simplest version of the classical symmetrization technique that applies to elliptic equations posed in a bounded domain or in the whole space. In this last case they may lose their uniform ellipticity as $|x| \rightarrow \infty$. Our modification replaces the standard (Schwarz) rearrangement by rearrangement with respect to a measure. 


\subsection{The problem}

Let us consider the equation

$$
-\frac{1}{\rho(x)} \sum_{i, j} \partial_{i}\left(a_{i j}(x) \partial_{j} u\right)=g(x, u)
$$

posed in the ball $\Omega=B_{R}(0)$, with boundary data $u=0$ in $\partial \Omega$. Though the reader may find it natural to move $\rho(x)$ to the right-hand side in order to simplify the notation, the application to parabolic equations makes the present form preferable. Here are the assumptions:

(H1) The coefficients $a_{i j}$ are locally bounded measurable functions in $\Omega$ satisfying the ellipticity hypothesis

$$
\sum_{i, j} a_{i j} \xi_{i} \xi_{j} \geq \lambda(x)|\xi|^{2}
$$

for some function $\lambda(x)>0$.

(H2) There is a bounded measurable, radially symmetric function $\rho_{0}$ and a constant $0<$ $c \leq 1$ such that

$$
c \rho_{0}(x) \leq \rho(x) \leq \rho_{0}(x) \quad \text { for } x \in \Omega .
$$

(H3) The function $g(x, u)$ is measurable, and

$$
g(x, u) u \leq f(x) u \quad \text { for a.e. } x \text { and all } u,
$$

where $f$ is a measurable function in some Lebesgue $L^{p}$ space, $1 \leq p \leq \infty \mathbb{1}$

\subsection{The symmetrized problem}

We need to define the symmetrized problem. We perform symmetrization of the function $f(x)$, defined in $\Omega$, with respect to the measure $d \mu=\rho(x) d x$ (cf. the definitions in Section 22. The symmetrized problem is then posed in the ball $\Omega_{\mu}^{*}=B_{R^{*}}(0)$ with Lebesgue measure $\int_{\Omega} \rho(x) d x$. The symmetrized equation will be

$$
-C \Delta_{y} \bar{u}=f_{\mu}^{*}(y) \quad \text { in } \Omega_{\mu}^{*},
$$

where $f_{\mu}^{*}$ is the $\mu$-spherical rearrangement of $f$ and $C$ is the constant described in Theorem 3.1. We take boundary conditions

$$
\bar{u}(y)=0 \quad \text { on } \partial \Omega_{\mu}^{*} .
$$

\subsection{Control of degeneracy and main result}

In order to obtain a comparison result we perform a change of variables $z=T(x)$ with the property

$$
\left.d z\right|_{T(x)}=\rho_{0}(x) d x=d \mu_{0}(x),
$$

\footnotetext{
1 Though other spaces also appear in the literature.
} 
where $\left.d z\right|_{T(x)}$ denotes the standard volume element in the $z$-space at the point $T(x)$. The main implication of 3.7 is that, given any function $f(x)$ and the corresponding function in the new variables $f(z)=f(x)$, we have

$$
\phi_{\tilde{f}}(k)=\phi_{f ; \mu_{0}}(k)
$$

and therefore $\mu_{0}$-symmetrization of $f$ is equivalent to standard symmetrization of $\tilde{f}$. In other words, the change of variables $z=T(x)$ can be seen as a first step towards weighted symmetrization. We use $\rho_{0}$ instead of $\rho$ at this stage for technical reasons, in particular because (3.7) can be achieved by means of a radial transformation $T$, i.e., in spherical coordinates $x=(r, \theta), z=(s, \psi)$ we will have $\theta=\psi \in \mathbb{S}^{n-1}$, and $s=s(r)$. Since the volume elements are given by $d z=s^{n-1} d s d \Omega_{n-1}$ and $d x=r^{n-1} d r d \Omega_{n-1} 2^{2}$ the function $s(r)$ is defined by the ODE

$$
s^{n-1} \frac{d s}{d r}=\rho_{0}(r) r^{n-1}
$$

plus the initial condition $s(0)=0$.

In order to prove our comparison result, we need to impose some conditions on the transformation $z=T(x)$ that are met under the following hypothesis:

(H4) If $n>1$, there exists a constant $K>0$ such that the functions $s(r), \rho_{0}(r)$ and $\lambda(x)$ satisfy

$$
s(r) \geq K r\left(\rho_{0}(r) / \lambda(x)\right)^{1 / 2}, \quad d s / d r \geq K\left(\rho_{0}(r) / \lambda(x)\right)^{1 / 2}
$$

for $x \in \Omega$ and $r=|x|$. This double condition can be simplified. With the help of (3.8), we arrive at the equivalent formulation

$$
K^{n}\left(\rho_{0} / \lambda\right)^{n / 2} \leq \frac{\int_{0}^{r} \rho_{0}(t) t^{n-1} d t}{r^{n} / n} \leq K^{-n /(n-1)}\left(\rho_{0} \lambda\right)^{n / 2(n-1)} .
$$

If $n=1$, we only require the second condition in 3.9 , which amounts to $K^{2} \leq$ $\rho_{0} \lambda$.

The main result is stated as follows. We use the weighted spaces $L^{2}(\Omega ; \rho)$ and $H_{0}^{1}(\Omega ; \lambda)$ defined in the standard way.

Theorem 3.1. Assume that $f \in L^{2}(\Omega ; \rho), f \geq 0$ and that $u \in H_{0}^{1}(\Omega ; \lambda)$ is a weak solution of equation (3.1) under the above hypotheses $(\mathrm{H} 1)-(\mathrm{H} 4)$. Then we can compare the $\mu$-symmetrization of $u$ with the solution of the symmetrized problem (3.5)-3.6 with constant $C=K^{2} c$ and obtain the pointwise result

$$
u_{\mu}^{*}(s) \leq \bar{u}(s) \quad \text { for all } s \in\left(0, R^{*}\right)
$$

\footnotetext{
${ }^{2} d \Omega_{n-1}$ is the surface element on the unit sphere $\mathbb{S}^{n-1}$; it will not appear later.
} 
Condition (H4) may look a bit abstract. We will give more specific sufficient conditions at the end of this section. These particular conditions also show the sharpness of our result.

Proof of Theorem 3.1] We follow the outline of the proof of the survey paper [V4] for the case $\rho=\lambda=1$ :

(i) Write equation (3.1) in variational form as

$$
\int_{\Omega} \sum_{i, j} a_{i j} \frac{\partial u}{\partial x_{i}} \frac{\partial v}{\partial x_{j}} d x=\int_{\Omega} g(x, u) \rho v d x
$$

for test functions $v \in H_{0}^{1}(\Omega ; \lambda)$. Since $f \geq 0$ we have $u \geq 0$ by the maximum principle. Let us now write $a(\nabla u, \nabla v) \equiv \sum a_{i j} \partial_{i} u \partial_{j} v$.

(ii) Let $\Omega(k)=\{u>k\}$. We calculate for a.e. $k>0$ the derivative $\frac{d}{d k} \int_{\Omega(k)} a(\nabla u, \nabla u) d x$. Taking as test function $v=(u-k)_{+}$in 3.12 we get

$$
\int_{\{u>k\}} a(\nabla u, \nabla u) d x=\int_{\{u>k\}} g(x, u) v(x) \rho d x .
$$

It is a classical result that for a.e. $k \in(0, \operatorname{ess} \sup (u))$ we have

$$
\frac{d}{d k} \int_{\{u>k\}} g v \rho d x=-\int_{\{u>k\}} g \rho d x .
$$

It follows that

$$
-\frac{d}{d k} \int_{\{u>k\}} a(\nabla u, \nabla u) d x=\int_{\{u>k\}} g(x, u) \rho d x .
$$

(iii) Put $Z(k, h)=\{x: k<u(x)<k+h\}$ for $k, h>0$. We recall that for every measurable function $F$ we have

$$
-\frac{d}{d k} \int_{\{u>k\}} F(x) d x=\lim _{h \rightarrow 0} \frac{1}{h} \int_{Z(k, h)} F(x) d x
$$

if the derivative exists. Combining the ellipticity assumption and 3.14 we conclude that

$$
-\frac{d}{d k} \int_{\{u>k\}} a(\nabla u, \nabla u) d x \geq-\frac{d}{d k} \int_{\{u>k\}} \lambda|\nabla u|^{2} d x \geq 0 .
$$

We transform in this way equality 3.13 into

$$
-\frac{d}{d k} \int_{\{u>k\}} \lambda|\nabla u|^{2} d x \leq \int_{\{u>k\}} g(x, u) \rho d x .
$$

(iv) We need to transform the left-hand side of the last formula. Using the CauchySchwarz inequality, we get

$$
\frac{1}{h} \int_{Z(k, h)} \lambda^{1 / 2}|\nabla u| \rho^{1 / 2} d x \leq\left(\frac{1}{h} \int_{Z(k, h)} \lambda|\nabla u|^{2} d x\right)^{1 / 2}\left(\frac{1}{h} \int_{Z(k, h)} \rho d x\right)^{1 / 2} .
$$


The first novelty of the calculation is the use of the weight $\rho(x)$. Thus, we can view the last integral as the measure of the set $Z(k, h)$ with respect to the measure $d \mu=\rho(x) d x$. We now consider the distribution function $\phi_{u ; \rho}(k)$ of the function $u$ with respect to this measure, as defined in Subsection 2.2, and we get in the limit $h \rightarrow 0$

$$
\begin{aligned}
\left(-\frac{d}{d k} \int_{\{u>k\}} \lambda^{1 / 2}|\nabla u| \rho^{1 / 2} d x\right)^{2} & \leq\left(-\frac{d}{d k} \int_{\{u>k\}} \lambda|\nabla u|^{2} d x\right)\left(-\phi_{u ; \rho}^{\prime}(k)\right) \\
& \leq\left(-\phi_{u ; \rho}^{\prime}(k)\right) \int_{\{u>k\}} g \rho d x
\end{aligned}
$$

by 3.15 .

(v) We now introduce the second novelty of the calculation, i.e., the change of coordinates $y=T(x)$ defined at the beginning of the subsection. Our goal is to absorb the factor $\rho^{1 / 2} \lambda^{1 / 2}$ on the left-hand side of 3.16 , thus obtaining an unweighted integral in the new variables. Toward this end, we need to transform the gradient with respect to $x$ into a gradient with respect to $z$; in spherical coordinates we have

$$
\frac{\partial u}{\partial r}=\frac{\partial \tilde{u}}{\partial s} \frac{d s}{d r}=\rho_{0}(r)(r / s)^{n-1} \frac{\partial \tilde{u}}{\partial s}
$$

and

$$
\frac{\partial u}{r \partial \theta}=\frac{s}{r} \frac{\partial \tilde{u}}{s \partial \theta} \quad \text { if } n>1 .
$$

Here, $\theta$ represents any direction on the unit sphere $\mathbb{S}^{n-1}$ for $n>1$. In this case, by (H2) and (H4), we have

$$
\begin{aligned}
K c^{1 / 2} \int_{Z_{0}(k, h)}\left|\nabla_{z} u\right| d z & \leq c^{1 / 2} \int_{Z_{0}(k, h)} \lambda^{1 / 2}\left|\nabla_{x} u\right| \rho_{0}^{-1 / 2} d z \\
& \leq \int_{Z(k, h)} \lambda^{1 / 2}\left|\nabla_{x} u\right| \rho^{1 / 2} d x
\end{aligned}
$$

where $Z_{0}(k, h)=\{z: k<\tilde{u}(z)<k+h\}$ and, by abuse of notation, we have denoted the functions $\tilde{u}(z)=u(x), \tilde{\lambda}(z)=\lambda(x)$, etc. simply by $u(z), \lambda(z)$ etc. Dividing by $h$, letting $h \rightarrow 0$ and recalling (3.14), 3.7) and (3.16), we get

$$
K^{2} c\left(-\frac{d}{d k} \int_{\{u>k\}}\left|\nabla_{z} u\right| d z\right)^{2} \leq\left(-\phi_{u ; \rho}^{\prime}(k)\right) \int_{\{u>k\}} g(x, u(x)) \rho(x) d x,
$$

where the notation $\{u>k\}$ on the left-hand side stands for the transformed set $\{z: \tilde{u}(z)$ $>k\}$. In the case $n=1,3.17$ and (3.18) reduce to

$$
\frac{d u}{d x}=\frac{d \tilde{u}}{d y} \frac{d y}{d x}=\rho_{0}(x) \frac{d \tilde{u}}{d y}
$$

and we are led to the same conclusion under (H4) for $n=1$. 
(vi) We now return to the standard proof and use two pieces of heavy artillery in order to estimate the left-hand side. First, Fleming-Rishel's formula says that for a.e. $k$

$$
P(\{u>k\})=-\frac{d}{d k} \int_{\{u>k\}}\left|\nabla_{z} u\right| d z,
$$

where $P$ denotes the perimeter and, as before, the set on the left-hand side is understood in the $z$-space. Then De Giorgi's isoperimetric inequality can be written as

$$
P(\{u>k\}) \geq n \omega_{n}^{1 / n} \phi_{u}(k)^{(n-1) / n}=n \omega_{n}^{1 / n} \phi_{u ; \rho_{0}}(k)^{(n-1) / n},
$$

where $\phi_{u}$ denotes the standard distribution function in the $z$-space, while $\phi_{u ; \rho_{0}}$ stands for the distribution function in the original $x$-space with respect to the measure $d \mu_{0}=$ $\rho_{0}(x) d x$, according to the notation introduced in Subsection 2.3. These functions coincide by the volume-preserving nature of our transformation $x \mapsto z$ (note that we always consider the Lebesgue measure when dealing with functions of the $z$-variable). If we use both formulas, 3.19 becomes

$$
C n^{2} \omega_{n}^{2 / n} \phi_{u ; \rho_{0}}(k)^{2-2 / n} \leq\left(-\phi_{u ; \rho}^{\prime}(k)\right) \int_{\{u>k\}} g(x, u(x)) \rho(x) d x, \quad C=K^{2} c .
$$

(vii) Moreover, by our assumptions on $g$,

$$
C n^{2} \omega_{n}^{2 / n} \phi_{u ; \rho_{0}}(k)^{2-2 / n} \leq\left(-\phi_{u ; \rho}^{\prime}(k)\right) \int_{\{u>k\}} f(x) \rho(x) d x .
$$

(viii) We now perform $\rho$-symmetrization of $f$. Using Hardy-Littlewood's inequality (2.11) we estimate

$$
\int_{\{u>k\}} f(x) \rho(x) d x \leq \int_{\left\{u_{\mu}^{*}>k\right\}} f_{\mu}^{*}(y) d y=\int_{B_{\sigma}} f_{\mu}^{*}(y) d y,
$$

where $\left.\left|B_{\sigma}\right|=\operatorname{meas}\left\{y: u_{\mu}^{*}(y)>k\right\}\right)=\phi_{u ; \rho}(k)$, i.e., $\omega_{n} \sigma^{n}=\phi_{u ; \rho}(k)$. Substituting into 3.24, we get the inequality

$$
C n^{2} \omega_{n}^{2 / n} \phi_{u ; \rho_{0}}(k)^{2-2 / n} \leq\left(-\phi_{u ; \rho}^{\prime}(k)\right) \int_{B_{\sigma}} f_{\mu}^{*}(y) d y .
$$

(ix) Next, recall that

$$
\phi_{u ; \rho_{0}}(k)=\mu_{0}(\{u>k\})=\int_{\{u>k\}} \rho_{0}(x) d x \geq \int_{\{u>k\}} \rho(x) d x=\mu(\{u>k\})=\phi_{u ; \rho}(k),
$$

by assumption (H2). Therefore, 3.25 implies

$$
C n^{2} \omega_{n}^{2 / n} \phi_{u ; \rho}(k)^{2-2 / n} \leq\left(-\phi_{u ; \rho}^{\prime}(k)\right) \int_{B_{\sigma}} f_{\mu}^{*}(y) d y .
$$


(x) At this stage we recall that 3.26 is satisfied with equality by the solution $\bar{u}$ of the symmetrized problem (3.5)-3.6. Indeed, we have

$$
-C n \omega_{n} \sigma^{n-1} \bar{u}^{\prime}(\sigma)=\int_{B_{\sigma}} f_{\mu}^{*}(y) d y .
$$

The comparison we are looking for follows easily since the equality $\phi_{u ; \rho}\left(u_{\mu}^{*}(\sigma)\right)=\omega_{n} \sigma^{n}$ for a.e. $\sigma$ implies that 3.26 can be written as

$$
-C n \omega_{n} \sigma^{n-1}\left(u_{\mu}^{*}\right)^{\prime}(\sigma) \leq \int_{B_{\sigma}} f_{\mu}^{*}(y) d y .
$$

Therefore, $\left(u_{\mu}^{*}\right)^{\prime}(\sigma) \leq \bar{u}^{\prime}(\sigma)$ for $\sigma<R^{*}$. Taking into account the boundary condition $u_{\mu}^{*}\left(R^{*}\right)=\bar{u}\left(R^{*}\right)=0$, we obtain the desired inequality.

Practical assumptions. As already remarked, condition (H4) is a bit abstract and we would like to obtain more specific conditions. When we deal with the Cauchy problem for equation (1.2), we are led to consider a sequence of problems posed in balls of increasing radius. If we assume that $\rho, \lambda>0$ in $\mathbb{R}^{n}$, condition (H4) clearly holds on each fixed ball $B_{R}$ with some $K=K(R)>0$. Therefore, in order to get a symmetrization result for the problem in the whole space, it is important to have simple conditions under which (H4) holds for arbitrarily large balls with a universal constant $K$. The following lemma deals with the case of power-like densities. It turns out that the result strongly depends on whether $\rho_{0} \in L^{1}\left(\mathbb{R}^{n}\right)$ or not, i.e., on whether the total mass of the medium is finite or not.

Lemma 3.2. Let $\rho_{0}(x)=A(1+|x|)^{-\alpha}$ with $A, \alpha>0$ and $\lambda(x) \sim|x|^{-\beta}$ as $|x| \rightarrow \infty$. Then condition (H4) holds on $B_{R}(0)$ for all $R>0$ with $K$ independent of $R$ if either

(i) $\alpha<n$ and $\alpha(n-2) \geq \beta n$, or

(ii) $\alpha=n$ and $\beta<n-2$, or

(iii) $\alpha>n$ and $\alpha+\beta \leq 2(n-1)$.

Proof. Boundedness from below of $\rho_{0}$ near $r=0$ implies that $s(r) \geq \bar{s}(r)$ for $r \sim 0$, where $\bar{s}(r)$ is the solution of the IVP

$$
s^{n-1} d s=C r^{n-1} d r, \quad s(0)=0,
$$

i.e., $\bar{s}(r)=C^{1 / n} r$. Then $s / r \geq C^{1 / n}$ and also $d s / d r \geq C^{1 / n}$ for $r \sim 0$. Next, we deal with $r \sim \infty$. From our hypotheses it easily follows that if $\alpha<n$ then

$$
s / r, d s / d r \sim r^{-\alpha / n} \quad \text { as } r \rightarrow \infty .
$$

Therefore, condition (H4) holds for $r \sim \infty$ if and only if

$$
\alpha(n-2) \geq \beta n .
$$

If $\alpha=n$, we have

$$
s / r \sim r^{-1} \log ^{1 / n} r, \quad d s / d r \sim r^{-1} \log ^{1 / n-1} r, \quad \text { as } r \rightarrow \infty .
$$


Consequently, (H4) holds for $r \sim \infty$ if and only if

$$
\beta<n-2 \text {. }
$$

Finally, if $\alpha>n$, we have

$$
s / r \sim r^{-1}, \quad d s / d r \sim r^{-\alpha+n-1}, \quad \text { as } r \rightarrow \infty,
$$

hence (H4) holds for $r \sim \infty$ if and only if

$$
\alpha \geq \beta+2 \text { and } \alpha+\beta \leq 2(n-1) .
$$

Remark 3.3. The situation is quite simple in 1D where the transformation $y=T(x)$ has only the radial direction and the necessary condition reduces to $\rho_{0}(x) \lambda(x) \geq K^{2}$. Indeed, in this case the weighted symmetrization result is not needed. Instead, we can work out the equivalent equation in $y$ variables. By means of $d y=\rho(x) d x$, we easily pass from

$$
-\frac{1}{\rho}\left(\lambda(x) u_{x}\right)_{x}=f(x)
$$

to the divergence equation

$$
-\left(\rho(x) \lambda(x) u_{y}\right)_{y}=f(y) .
$$

Now, if $\rho(x) \lambda(x) \geq C>0$ the standard symmetrization result applies. This coincides with the condition above if (H2) holds. In Lemma 3.2 the condition is $\alpha+\beta \leq 0$, which also coincides with this analysis in the power-like case $\rho(x) \sim|x|^{-\alpha}, \lambda(x) \sim|x|^{-\beta}$ as $|x| \rightarrow \infty$.

Remark 3.4. Observe that, for $n=1$, a decreasing $\rho$ is allowed only if $\lambda(x)$ grows at infinity. For $n=2, \lambda(x)$ must be bounded from below. Finally, for $n \geq 3$, we may allow decreasing $\lambda(x)$ on the condition that $\rho$ decreases at least with a matching rate.

Remark 3.5. In the particular case $\beta=0$ it follows that (H4) holds if and only if $n=2$ and $0<\alpha<2$, or $n \geq 3$ and $0<\alpha \leq 2(n-1)$.

\section{Symmetrization and comparison. Elliptic problems II}

We now deal with the additional consequences of having a lower-order term in the equation. This is motivated by the parabolic application in the next section. Here, we review the basic theory since it leads at the end of the section to the presentation of the interaction between both techniques. Such interaction needs a different way of looking at the standard symmetrization inequality in terms of concentration comparison. To be specific, we consider again the Dirichlet problem in the ball $\Omega$ but now for the equation with a more specialized structure

$$
-\frac{1}{\rho(x)} \sum_{i, j} \partial_{i}\left(a_{i j}(x) \partial_{j} u\right)+b(x, u)=f .
$$

Previous assumptions (H1)-(H4) on $a_{i j}$ and $\rho$ remain in force. Concerning the function $b(x, u)$, we assume it is measurable, continuous and nondecreasing in $u$ for fixed $x$, and 
bounded in $x$ uniformly for bounded $u$. Moreover, we assume that

$$
b(x, u) u \geq 0 \quad \text { for a.e. } x \text { and all } u .
$$

The right-hand side $f$ is a measurable function in some Lebesgue $L^{p}$ space.

In the preceding section the effect of the zero-order term has been neglected through the assumption (3.4), which is equivalent to (4.2) in the particular case of (3.1). However, this leads to a poorer understanding and poorer estimates. This subject has been investigated by a number of authors. Briefly stated, the problem is that keeping track of this term changes the last part of the preceding proof and forces us to change the type of comparison in the conclusion. We have been first led to keeping track of the term in the study of parabolic problems by implicit discretization in time (see Section 5). It turns out that, in the spirit of the end of the previous proof, there is a simple modification that naturally leads to concentration comparison, which is a weaker result, but enough for our purposes. In this way we can compare the result of solving and then rearranging with the result of the reverse procedure, i.e., first rearranging and then solving the symmetrized problem. This is our main result.

Theorem 4.1. In addition to the assumptions of Theorem 3.1 on $f$ and $u$, assume

(H5) $b(x, w) w \geq \beta(w) w$ for all $w$,

where $\beta$ is a nondecreasing function with $\beta(0)=0$. Put $v=\beta(u)$. Let $\bar{u}(s), 0<s<R^{*}$, be an integral supersolution of the radial problem

$$
-C \Delta \bar{u}+\beta(\bar{u})=\bar{f}(s),
$$

with boundary condition $\bar{u}\left(R^{*}\right) \geq 0$, where $\bar{f}$ is a radial function in $L^{1}\left(\Omega_{\mu}^{*}\right)$ such that $\bar{f} \succ f^{*}$. Put $\bar{v}(s)=\beta(\bar{u})$. Then the two radial functions $v_{\mu}^{*}(s)$ and $\bar{v}(s)$ are ordered in the sense of concentration:

$$
v_{\mu}^{*} \prec \bar{v} .
$$

Proof. We repeat the previous proof with $g(x, u)=f-b(x, u)$. Using (H5), we arrive at formula (3.24) with right-hand side replaced by

$$
\left(-\phi_{u ; \rho}^{\prime}(k)\right) \int_{\{u>k\}}[f(x)-\beta(u)] \rho(x) d x \leq\left(-\phi_{u ; \rho}^{\prime}(k)\right) \int_{B_{\sigma}}\left[f_{\mu}^{*}(y)-\beta\left(u_{\mu}^{*}\right)\right] d y,
$$

where we have used the conservation of integrals (2.8), the Hardy-Littlewood inequality 2.11 and the obvious fact that $[\beta(u)]_{\mu}^{*}=\beta\left(u_{\mu}^{*}\right)$. Thus, we arrive at

$$
C n^{2} \omega_{n}^{2 / n} \phi_{u ; \rho_{0}}(k)^{2-2 / n} \leq\left(-\phi_{u ; \rho}^{\prime}(k)\right) \int_{B_{\sigma}}\left[f_{\mu}^{*}(y)-\beta\left(u_{\mu}^{*}\right)\right] d y .
$$

Arguing as in step (ix) of the proof of Theorem 3.1 we conclude that

$$
C n^{2} \omega_{n}^{2 / n} \phi_{u ; \rho}(k)^{2-2 / n} \leq\left(-\phi_{u ; \rho}^{\prime}(k)\right) \int_{B_{\sigma}}\left[f_{\mu}^{*}(y)-\beta\left(u_{\mu}^{*}\right)\right] d y .
$$


As in step (x) of that proof, we observe that this last inequality can be written as

$$
-C n \omega_{n} \sigma^{n-1}\left(u_{\mu}^{*}\right)^{\prime}(\sigma) \leq \int_{B_{\sigma}}\left[f_{\mu}^{*}(y)-\beta\left(u_{\mu}^{*}\right)\right] d y,
$$

which is nothing else than the integrated version of the inequality

$$
-C \Delta u+\beta(u) \leq f_{\mu}^{*} .
$$

In the terminology of Section $2, u_{\mu}^{*}(s)$ is a radial integral subsolution of the equation

$$
-C \Delta u+\beta(u)=f_{\mu}^{*}
$$

in the ball $\Omega_{\mu}^{*}$. By assumption, $\bar{u}$ is a solution (hence a radial integral solution) of the same equation, maybe with more concentrated right-hand side. Comparison is now a consequence of Theorem 2.4. The fact that we are in the standard case is ensured by the Dirichlet conditions $u=0$ on $\partial \Omega$ (and hence $u_{\mu}^{*}=0$ on $\partial \Omega_{\mu}^{*}$ ) and the nonnegative condition for $\bar{u}$.

The concentration statement can be reformulated in terms of standard norms by means of Lemma 2.2

Corollary 4.2. Let the assumptions of Theorem 4.1 hold. Then for every convex nondecreasing function $\Phi:[0, \infty) \rightarrow[0, \infty)$ with $\Phi(0)=0$ we have

$$
\int_{\Omega_{\mu}^{*}} \Phi\left(v_{\mu}^{*}(y)\right) d y \leq \int_{\Omega_{\mu}^{*}} \Phi(\bar{v}(y)) d y .
$$

In particular, for every $1 \leq p \leq \infty$ we have

$$
\|v\|_{L^{p}(\Omega ; \rho)}=\left\|v_{\mu}^{*}\right\|_{L^{p}\left(\Omega_{\mu}^{*}\right)} \leq\|\bar{v}\|_{L^{p}\left(\Omega_{\mu}^{*}\right)} .
$$

\section{Symmetrization for parabolic problems}

In order to treat nonlinear, possibly degenerate equations like

$$
\rho(x) u_{t}=\sum_{i, j} \partial_{i}\left(a_{i j}(x) \partial_{j} \varphi(u)\right)+f \rho,
$$

the survey paper [V1] proposes (for $\rho=1$ and uniformly elliptic matrix $a_{i j}$ ) to use the technique of Implicit Time Discretization (ITD for short); in this way the original problem of obtaining a priori estimates for equation (5.1) is reduced to obtaining similar estimates for elliptic equations of a definite type. In fact, by replacing the time derivative by an increment quotient and using a partition of the time interval $[0, T]$ of the form $t_{0}=0<t_{1}<\cdots<t_{N}=T$, we are reduced to solving a sequence of elliptic problems with zero-order term of the form

$$
-h_{k} \sum_{i, j} \partial_{i}\left(a_{i, j}(x) \partial_{j} v\left(t_{k}\right)\right)+\rho u\left(t_{k}\right)=\rho u\left(t_{k-1}\right)+h \rho f\left(t_{k-1}\right), \quad v\left(t_{k}\right)=\varphi\left(u\left(t_{k}\right)\right) .
$$

By means of this scheme we compute the value $u\left(t_{k}\right)$ of the discretized solution at time $t_{k}$ in terms of the value of $u$ in the previous step, $u\left(t_{k-1}\right)$. Here, $k$ runs from 1 to $N$, 
$h_{k}=t_{k}-t_{k-1}>0$, and $f_{k}=f_{k}(x)$ is a suitable discretization of the function $f=f(x, t)$ at the mesh times; the notation $u\left(t_{k}\right)$ means $u\left(x, t_{k}\right)$, seen as a function of $x$ for fixed $t=t_{k}$, and likewise $v\left(t_{k}\right)$; more precisely, $v\left(t_{k}\right)$ is related to $u\left(t_{k}\right)$ by $v(x, t)=\varphi\left(u\left(x, t_{k}\right)\right)$ a.e. in $x$. Therefore, each step reduces to solving the elliptic equation

$$
-\frac{h}{\rho(x)} \Delta \varphi(u)+u=F,
$$

equivalently,

$$
-\frac{h}{\rho(x)} \Delta v+\beta(v)=F,
$$

where $\beta=\varphi^{-1}$ and $F(x)$ is a different, but known function in each step. For $t_{k-1}<t$ $<t_{k}$, we make linear interpolation, thus producing an approximate solution $u_{N}(x, t)$. The convergence $u_{N} \rightarrow u$ follows from the famous

Theorem 5.1 (Crandall-Liggett Theorem [CL]). Let A be an m-accretive operator in the Banach space $X$ with domain $\mathcal{D}(A)$. Then, for any $u_{0} \in \overline{\mathcal{D}(A)}$,

$$
e^{-t A} u_{0}=\lim _{n \rightarrow \infty}\left(J_{t / n}\right)^{n} u_{0}
$$

exists uniformly on compact subsets of $\left[0, \infty\left[\right.\right.$. Moreover, the family of operators $e^{-t A}$, $t>0$, is a continuous semigroup of contractive mappings of $\overline{\mathcal{D}(A)}$.

We choose the space $X=L^{1}(\Omega ; \rho)$, and as $A$ a suitably defined differential operator. We take as $\mathcal{D}(A)$ the set of functions $u \in X$ such that $\varphi(u) \in W_{0}^{1,1}(\Omega)$. We then define

$$
A(u)=-\frac{1}{\rho(x)} \Delta \varphi(u) .
$$

Under the condition that $\rho(x)$ is positive and bounded, $A$ defined by formula 5.6 is $m$ accretive; proving this amounts to solving $-h \Delta \varphi(u)+\rho(x) u=\rho(x) F$ so that the map $F \mapsto u$ is contractive in $X$ (cf. [BrS, $, \mathrm{GM}, \overline{\mathrm{BG}}])$.

The theorem above provides the existence of a mild (or semigroup) solution to the Cauchy problem for (5.1) with $u_{0} \in X$. In the following, by solution to (5.1) we shall always mean the mild solution with a definition of the operator $A$ that makes it $m$ accretive in $X$. Combining the Crandall-Liggett generation theorem with Theorem 4.1 we can prove the following

Theorem 5.2. Let $u$ be the mild solution of the initial-boundary value problem for the equation (5.1) with data $u_{0} \in L^{1}(\Omega ; \rho), u=0$ in $\partial \Omega$, and second member $f \in L^{1}(\Omega ; \rho)$ under the assumptions $(\mathrm{H} 1)-(\mathrm{H} 5)$ on $\rho(x)$ and $a_{i j}(x)$. Let $\bar{u}$ be the solution of the problem

$$
u_{t}=\Delta \bar{\varphi}(u)+g(s, t), \quad u(y, 0)=\bar{u}_{0}(s),
$$

posed in the ball $\Omega_{\mu}^{*}$, where $\bar{\varphi}=C \beta^{-1}$ with radially symmetric data $\bar{u}_{0} \in L^{1}\left(\Omega_{\mu}^{*}\right)$, $\bar{u}_{0}(s) \geq 0$ and (spatially) radially symmetric right-hand side $g(s, t) \geq 0$. Assume moreover that

(i) $u_{0, \mu}^{*} \prec \bar{u}_{0}$,

(ii) $f_{\mu}^{*}(\cdot, t) \prec g(\cdot, t)$ for every $t \geq 0$. 
Then, for every $t \geq 0$,

$$
u_{\mu}^{*}(\cdot, t) \prec \bar{u}(\cdot, t) .
$$

Proof. Using the Crandall-Liggett result we are reduced to comparing the discretization steps, which consist of elliptic problems as those treated in Sections 3 and 4 It is important to realize that comparison of concentrations between the discretized versions of the solutions is inherited in every step of the iteration. We proceed as follows. In the first step, between $t_{0}=0$ and $t_{1}=t / N$, we start from a datum $u_{0}$ and a forcing term $f_{0}$, and obtain a solution of the elliptic problem (5.2)

$$
-h \sum_{i, j} \partial_{i}\left(a_{i, j}(x) \partial_{j} \phi(u)\right)+\rho u=\rho\left(u_{0}+h f_{0}\right),
$$

which is a form of 4.1. Let us call the solution $u_{1}$. We symmetrize it into $u_{1, \mu}^{*}$, defined in $\Omega_{\mu}^{*}$, and it becomes a $\prec$-subsolution of the symmetric problem 4.3 with right-hand side $u_{0, \mu}^{*}+h f_{0, \mu}^{*}$. Note that this second member is more concentrated than $\left(u_{0}+h f_{0}\right)_{\mu}^{*}$. We compare this solution with the radially symmetric solution $\bar{u}_{1}$ of the homogeneous elliptic equation appearing in the first iteration step for the symmetrized parabolic problem (5.7) with data $\bar{u}_{0}+h g_{0}$. By Theorem 4.1, we get

$$
u_{1, \mu}^{*} \prec \bar{u}_{1} \text {. }
$$

In the second step we have to solve an elliptic problem three times: the first elliptic equation with data $u_{1}+h f_{1}$ to get the second step of the discretized solution, $u_{2}$; the symmetrized version with data $u_{1, \mu}^{*}+h f_{1, \mu}^{*}$ to get a radial solution $w_{2}$; and the symmetrized problem 5.7 with $\bar{u}_{1}+h g_{1}$ to get a radial solution $\bar{u}_{2}$. The same type of comparison gives

$$
u_{2, \mu}^{*} \prec w_{2} \prec \bar{u}_{2} .
$$

The process is then continued for all the steps. Therefore, the comparison of concentrations works at all levels. To end the proof, the limit is taken as the time-step length goes to 0 .

Corollary 5.3. In particular, under the assumptions of Theorem 2.4 for every $t \geq 0$ and every $p \in[1, \infty]$ we have comparison of $L^{p}$ norms,

$$
\|u(\cdot, t)\|_{L^{p}(\Omega ; \rho)}=\left\|u_{\mu}^{*}(\cdot, t)\right\|_{L^{p}\left(\Omega_{\mu}^{*}\right)} \leq\|\bar{u}(\cdot, t)\|_{L^{p}\left(\Omega_{\mu}^{*}\right)} .
$$

Note that the terms of (5.9) can also be infinite for some or all values of $p$.

\section{Smoothing effects for the inhomogeneous porous medium equation}

In this section, we denote $L^{p}\left(\mathbb{R}^{n} ; \rho\right)$ simply by $L_{\rho}^{p}$. As an application of our previous results, in this section we prove sharp $L^{p}$ estimates (including $p=\infty$ ) for the solutions to the Cauchy problem for the inhomogeneous porous medium equation

$$
\left\{\begin{array}{l}
\rho(x) u_{t}=\Delta u^{m}, \\
u(x, 0)=u_{0},
\end{array}\right.
$$


in the slow diffusion case $m>1$, posed for $x \in \mathbb{R}^{n}, t>0$. The basic theory for problem 6.1 with $\rho \in C^{1}(\mathbb{R})$ and bounded continuous data $u_{0}$ is developed in [KR1], [KR2], [E], $[\overline{\mathrm{GHP}}$. A minimal solution is obtained as a monotone limit of solutions to the problems

$$
\left\{\begin{array}{l}
\rho(x) u_{t}=\Delta u^{m}, \\
u(x, 0)=u_{0 k} \quad \text { on }\{|x| \leq k\}, \\
u(x, t)=0 \text { on }\{|x|=k\},
\end{array}\right.
$$

where $u_{0 k} \uparrow u_{0}$ uniformly on compact subsets of $\mathbb{R}^{n}$. Uniqueness of weak solutions for $n=1,2$ is proved in [KR1] for general bounded $\rho \in C^{1}$ and bounded smooth data. In [GHP], an $L_{\rho}^{1}$-contractivity result and consequent uniqueness in the natural class $L_{\rho}^{1}$ is proved, again in dimensions $n=1,2$. It should be mentioned that in this last paper no smoothness of $\rho$ is assumed, but merely $\rho \in C\left(\mathbb{R}^{n}\right)$.

We shall call the integral $\int_{\mathbb{R}^{n}} u(x, t) \rho(x) d x=\|u(t)\|_{L_{\rho}^{1}}$ the energy of the solution $u$ at time $t$. An interesting question is to determine under which conditions the energy is preserved in time, which always happened in the homogeneous case for $m \geq 1$. Theorem 6.1 gives some information in this respect (see Remark 6.4).

In what follows, by solution to 6.1 we always mean the minimal solution in the above sense.

Theorem 6.1. Let $\rho \in C^{1}(\mathbb{R})$ and

$$
c_{2}(1+|x|)^{-\alpha} \leq \rho(x) \leq c_{1}(1+|x|)^{-\alpha}, \quad c_{1}, c_{2}>0 .
$$

Let either $n=2$ and $0<\alpha<2$, or $n \geq 3$ and $0<\alpha \leq 2(n-1)$. Let $u$ be the weak solution to 6.1 with $u_{0} \in L_{\rho}^{1} \cap L^{\infty} \cap \bar{C}\left(\mathbb{R}^{n}\right), u_{0} \geq 0$. Then $u(\cdot, t) \in L^{\infty}\left(\mathbb{R}^{n}\right)$ for all $t>0$ and we have an estimate of all $L_{\rho}^{p}$-norms for $1 \leq p \leq \infty$. There are two cases:

(i) If $\alpha \leq n$ so that the mass of the medium is infinite, we have the estimates

$$
u(x, t) \leq C t^{-n /(n(m-1)+2)}, \quad\|u(\cdot, t)\|_{L_{\rho}^{1}} \leq\left\|u_{0}\right\|_{L_{\rho}^{1}},
$$

where $C=C\left(\left\|u_{0}\right\|_{L_{\rho}^{1}}, c_{1}, c_{2}, m, n\right)$.

(ii) If $\alpha>n$ so that the mass of the medium is finite, then the energy of the solution decreases in time and we have the estimates

$$
u(x, t) \leq C t^{-1 /(m-1)}, \quad\|u(\cdot, t)\|_{L_{\rho}^{1}} \leq C^{\prime} t^{-1 /(m-1)},
$$

where $C$ and $C^{\prime}$ depend on $\left\|u_{0}\right\|_{L_{\rho}^{1}}, c_{1}, c_{2}, m$, and $n$.

Proof. We will apply the results of Theorem 4.1 to the approximate problems 6.2 , with $f=g=0, a_{i j}=\delta_{i j}, \varphi(w)=w^{m}$ and $\rho_{0}(x)=(1+|x|)^{-\alpha}$. Since $\rho>0$ in $\Omega_{k}$, the operator $[5.6$ is $m$-accretive, thanks to the results in [BrS]. In this case, the mild solution to 6.2 coincides with the weak solution. Moreover, by Remark 3.5. under our hypotheses on $\alpha$ conditions (H1)-(H5) hold with $K$ independent of $k$. Therefore,

$$
u_{k, \mu}^{*}(\cdot, t) \prec \bar{u}_{k}(\cdot, t),
$$


where $\bar{u}_{k}$ is the solution to the problem with constant diffusivity

$$
\left\{\begin{array}{l}
u_{t}=C \Delta u^{m}, \\
u(y, 0)=u_{0 k, \mu}^{*} \quad \text { on }\left\{|y| \leq R_{k}\right\}, \\
u(y, t)=0 \quad \text { on }\left\{|y|=R_{k}\right\},
\end{array}\right.
$$

with $R_{k}$ such that meas $\left(B_{R_{k}}(0)\right)=\mu\left(B_{k}(0)\right)$ and $C$ independent of $k$. Since $\left\{u_{0 k}\right\}$ is increasing, so is $\left\{u_{0 k, \mu}^{*}\right\}$. When passing to the limit $k \rightarrow \infty$, we should distinguish two cases:

(i) If $\rho \notin L^{1}$, i.e., if $\alpha \leq n$, we have $R_{k} \uparrow \infty$. In this case, we will have

$$
u_{\mu}^{*}(\cdot, t) \prec \bar{u}(\cdot, t),
$$

where $\bar{u}$ is the solution of the Cauchy problem

$$
\left\{\begin{array}{l}
\bar{u}_{t}=C \Delta_{y} \bar{u}^{m}, \\
\bar{u}(y, 0)=u_{0, \mu}^{*} \quad \text { on } \Omega_{\mu}^{*}=\mathbb{R}_{y}^{n} .
\end{array}\right.
$$

Since $u_{0, \mu}^{*} \in L^{1}$, for problem 6.5 standard smoothing results [V5] apply. In particular, we know that $\bar{u}(t) \in L^{\infty}$ for every $t>0$ and moreover

$$
\bar{u}(\cdot, t) \leq c(C, m, n)\left\|\bar{u}_{0}\right\|_{L^{1}}^{\sigma} t^{-\gamma}
$$

with

$$
\gamma=\frac{n}{n(m-1)+2}, \quad \sigma=\frac{2 \gamma}{n} .
$$

The assertion of the theorem in this case follows now from 6.4, 6.6, Lemma 2.2 and (2.10).

(ii) $\rho \in L^{1}$, i.e., $\alpha>n$. Then $R_{k} \uparrow R<\infty$, with meas $\left(B_{R}(0)\right)=\mu\left(\mathbb{R}^{n}\right)$. Therefore, $\Omega_{\mu}^{*}=B_{R}(0)$ and the limit problem is

$$
\left\{\begin{array}{l}
\bar{u}_{t}=C \Delta_{y} \bar{u}^{m}, \\
\bar{u}(y, 0)=u_{0, \mu}^{*} \quad \text { on } \Omega_{\mu}^{*}, \\
\bar{u}(y, t)=0 \quad \text { on }|y|=R .
\end{array}\right.
$$

For this last problem, we know the estimate

$$
\bar{u}(\cdot, t) \leq c(m, n)(C t)^{-1 /(m-1)}
$$

(see $[\mathrm{AP}],[\mathrm{V} 3])$. Arguing as before, the assertion follows for this case.

Remark 6.2. Much in the same way, one can give estimates in $L_{\rho}^{p}$ for $p \in[1, \infty)$. Indeed, by $L_{\rho}^{1}-L^{\infty}$ interpolation, we obtain $u(\cdot, t) \in L_{\rho}^{p}$ for $t>0$ and

$$
\|u(\cdot, t)\|_{L_{\rho}^{p}} \leq\|u(\cdot, t)\|_{L^{\infty}}^{(p-1) / p}\|u(\cdot, t)\|_{L_{\rho}^{1}}^{1 / p} \leq C_{p} t^{-\sigma_{p}},
$$

where $\sigma_{p}=-n(p-1) / p(n(m-1)+2)$ if $\alpha \leq n$ and $\sigma_{p}=1 /(m-1)$ if $\alpha>n$. 
Remark 6.3. Eidus $[\mathrm{E}]$ proves decay estimates of solutions to 6.1 in the space $L_{\rho}^{m+1}$ for $\alpha>n \geq 3$. Our decay rates agree with his, but the constant in $[\mathrm{E}]$ depends on $\left\|u_{0}\right\|_{\infty}$, while in our case it depends only on $\left\|u_{0}\right\|_{L^{1}}$. Also, our estimates for $\alpha>2$ follow from the results in $[\overline{\mathrm{BrK}}]$, actually the results in $[\mathrm{BrK}]$ are sharper in this range. However, our results are new for $\alpha<2$. We thank the referee for pointing out these facts.

Remark 6.4. For $n=2$ and $u_{0}$ as in the theorem, the energy $\|u(\cdot, t)\|_{L_{\rho}^{1}}$ is preserved in time (see $[\overline{\mathrm{GHP}}]$ ). For $\alpha>2$, this gives rise to isothermalization, hence no decay takes place. In contrast, for $n \geq 3$ and $\alpha>n$ (hence $\rho \in L^{1}$ ), it is proved in [KK] that $\|u(\cdot, t)\|_{L_{\rho}^{1}}$ decays. Theorem 6.1 provides an alternative proof of this fact for $\alpha \in$ $(n, 2(n-1)]$, as well as a quantitative estimate of the energy loss.

Remark 6.5. The estimates above are not true for the Barenblatt-type solutions considered in [KR1], since they solve a problem with singular density $\rho(x)=|x|^{-\alpha}$. Such a density modifies the decay rates near $t=0$.

\subsection{Counterexample in one dimension}

In this subsection we construct a solution to 6.1 for $n=1$ with $u_{0} \in L_{\rho}^{1}$ and $u(\cdot, t) \notin$ $L^{\infty}$ for small $t>0$. We assume that $\rho \in C^{1}(\mathbb{R})$ and

$$
\rho(x)=\rho_{k}:=2^{-\alpha k} \quad \text { on }\left[2^{k}-2^{k-2}, 2^{k}+2^{k-2}\right] \cup\left[-2^{k}-2^{k-2},-2^{k}+2^{k-2}\right]
$$

for $k=0,1, \ldots$ and some $\alpha>0$ to be chosen later.

Take $u_{0}=\sum_{k=1}^{\infty} u_{0 k}$, where $u_{0 k}(x)=U_{k}(x, 1)$ and $U_{k}$ is the Barenblatt solution of the homogeneous problem

$$
\left\{\begin{array}{l}
\rho_{k} u_{t}=\left(u^{m}\right)_{x x}, \\
u\left(x_{k}, 0\right)=M_{k} \delta\left(x-x_{k}\right),
\end{array}\right.
$$

where $x_{k}=2^{k}$ and $k=0,1,2, \ldots$ The masses $M_{k}=D x_{k}^{\beta}$ are chosen in such a way that the supports of $u_{0 k}$ are disjoint. Taking into account that the factor $\rho_{k}$ in 6.9 can be absorbed by introducing a new time $t^{\prime}=t / \rho_{k}$ and the explicit formulas for the Barenblatt solutions, we have

$$
\operatorname{supp} U_{k}(1)=\left[x_{k}-C M_{k}^{(m-1) /(m+1)} t^{\prime 1 /(m+1)}, x_{k}+C M_{k}^{(m-1) /(m+1)} t^{\prime 1 /(m+1)}\right],
$$

where $t^{\prime}=\rho_{k}^{-1}$ and $C=C(m)$. Then we require $C M_{k}^{(m-1) /(m+1)} t^{\prime 1 /(m+1)} \leq 2^{k-3}$, which amounts to asking

$$
D C^{\prime} 2^{k(\beta(m-1)+\alpha-(m+1)) /(m+1)} \leq 1,
$$

where $C^{\prime}=C^{\prime}(m, \alpha)$. For this to hold it is enough to take $D$ small and

$$
\beta(m-1)+\alpha \leq m+1 .
$$


On the other hand, we want $\int \rho u_{0} d x<\infty$. This requirement is equivalent to

$$
\sum_{k=0}^{\infty} \rho_{k} M_{k}<\infty
$$

i.e., $\sum 2^{(\beta-\alpha) k}<\infty$. Thus, we need $\beta<\alpha$. Finally, we require $u_{0} \notin L^{\infty}$. This holds if $\max U_{k}(x, 1) \rightarrow \infty$ as $k \rightarrow \infty$. But

$$
\max U_{k}(x, 1)=U_{k}\left(x_{k}, 1\right)=C(m) M_{k}^{2 /(m+1)}=C(m) 2^{2 k \beta /(m+1)},
$$

therefore we need $\beta>0$. The three conditions above on $\alpha$ and $\beta$ are met with some $\beta$ for any given $\alpha<m+1$. Our next goal is to show that $u(\cdot, t) \notin L^{\infty}$ for $t>0$ small. First we observe that there exist $\tau>0$ such that supp $U_{k}(1+\tau) \subset\left(2^{k}-2^{k-2}, 2^{k}+2^{k-2}\right)$ are disjoint and that $\max U_{k}(x, 1+\tau)=U_{k}\left(x_{k}, 1+\tau\right) \rightarrow \infty$ as $k \rightarrow \infty$. Actually, these two assertions follow exactly as above for $\tau=0$, once the requirements on $\alpha$ and $\beta$ are met. Finally, we observe that $u(x, \tau)=\sum U_{k}(x, 1+\tau)$, since $\rho(x)=\rho_{k}$ on $\left[2^{k}-2^{k-2}, 2^{k}+2^{k-2}\right]$. This ends the construction.

Note that this solution belongs to $C\left([0, \tau), L_{\rho}^{1}\right)$ and is strong in the sense that $u, u_{t},\left(u^{m}\right)_{x x} \in L_{\text {loc }}^{1}$. It is also the mild solution of the problem since each of the components is, hence every finite sum is (since they have separate supports), and finally mild solutions depend continuously on the data in the $L_{\rho}^{1}$ norm.

\section{Appendix}

This appendix is devoted to the proof of the weighted Hardy-Littlewood inequality 2.11 with a nonnegative absolutely continuous measure $\mu$.

Theorem 7.1. Let $(\Omega, \mu)$ be a measure space with $\Omega \subset \mathbb{R}^{n}$ and $\mu$ a nonnegative, absolutely continuous measure. Let $f$ be a $\mu$-measurable function and let $f_{\mu}^{*}$ denote its $\mu$-symmetrization on the ball $\Omega_{\mu}^{*}$, as defined in Subsection 2.3. Then for every ball $B_{R}(0) \subset \Omega_{\mu}^{*}$ we have

$$
\int_{B_{R}(0)} f_{\mu}^{*} d y=\sup \left\{\int_{E}|f| d \mu: E \subset \Omega, \mu(E) \leq \operatorname{meas}\left(B_{R}\right)\right\} .
$$

Proof. Fix a $\mu$-measurable $E \subset \Omega$ with $\mu(E) \leq \operatorname{meas}\left(\Omega_{\mu}^{*}\right)$. Define $\Omega_{k}=\{x \in \Omega$ : $|f(x)|>k\}$. The monotonicity of the distribution function implies that there are two possibilities.

(a) There exist $k \geq 0$ and a measurable set $C$ with $f=k$ a.e. on $C$ such that $\mu\left(\Omega_{k} \cup C\right)=$ $\mu(E)$. Then we have

$$
\begin{aligned}
\int_{E}|f| d \mu & =\int_{E \cap\left(\Omega_{k} \cup C\right)}|f| d \mu+\int_{E \backslash\left(\Omega_{k} \cup C\right)}|f| d \mu \\
& \leq \int_{E \cap\left(\Omega_{k} \cup C\right)}|f| d \mu+\int_{\left(\Omega_{k} \cup C\right) \backslash E}|f| d \mu \\
& =\int_{\Omega_{k} \cup C}|f| d \mu=\int_{B_{R}(0)} f_{\mu}^{*} d x
\end{aligned}
$$


with meas $\left(B_{R}(0)\right)=\mu\left(\Omega_{k} \cup C\right)=\mu(E)$, since $|f| \leq k$ in $E \backslash\left(\Omega_{k} \cup C\right),|f| \geq k$ in $\left(\Omega_{k} \cup C\right) \backslash E$ and $\mu\left(E \backslash\left(\Omega_{k} \cup C\right)\right)=\mu\left(\left(\Omega_{k} \cup C\right) \backslash E\right)$. In this case the supremum is achieved at $E=\Omega_{k} \cup C$. Note that $C$ is needed to take into account possible sets where $f$ is flat, so that the distribution function is discontinuous. Generically, those sets have zero measure and need not be considered.

(b) $\mu\left(\Omega_{0}\right)<\mu(E)$. Then

$$
\int_{E}|f| d \mu=\int_{E \cap \Omega_{0}}|f| d \mu .
$$

Since $E \cap \Omega_{0} \subseteq \Omega_{0}$, there exist $k \geq 0$ and a measurable set $C$ with $f=k$ a.e. on $C$ such that $\mu\left(\Omega_{k} \cup C\right)=\mu\left(E \cap \Omega_{0}\right)$ and we conclude as above:

$$
\int_{E}|f| d \mu \leq \int_{\Omega_{k} \cup C}|f| d \mu=\int_{B_{R}^{\prime}(0)} f_{\mu}^{*} d x \leq \int_{B_{R}(0)} f_{\mu}^{*} d x
$$

with meas $\left(B_{R}^{\prime}(0)\right)=\mu\left(E \cap \Omega_{0}\right)$ and meas $\left(B_{R}(0)\right)=\mu(E)$ (hence $\left.R^{\prime} \leq R\right)$. In this case the supremum is achieved at any set $E^{\prime} \supset \Omega_{0}$ with $\mu\left(E^{\prime}\right)=\mu(E)$, since $f_{\mu}^{*}=0$ a.e. in the annulus $R^{\prime}<|y|<R$.

\section{Final comments}

The topics of rearrangement and symmetrization are covered in many classical texts; for more details, we refer e.g. to the books $[\overline{\mathrm{Ba}} \mid,[\mathrm{BS}],[\mathrm{Kw}],[\mathrm{LL}]$, or the articles $[\mathrm{W}]$, [T1]-[T3]. Symmetrization with weights is studied by several authors, like Talenti [T4]. The concept of symmetrization with respect to a measure that we use is asymmetric with respect to the spaces, in the sense that we pass from a space with a measure given by a weight to a space endowed with the plain Lebesgue measure, where the model problem is posed and solved. This seems to be the best option for the comparison results we were aiming at. Such a concept has been studied by Vera de Serio in her thesis (see [SVS]). There are other options for symmetrization with weights in the literature like the ones using Gaussian measures (on both sides, cf. [BBMP]$]$ ).

The a priori estimates obtained in this paper are a useful tool in elaborating a theory of solutions of the inhomogeneous PME 5.1) with data in $L_{\rho}^{1}$. This will be the object of a separate publication [RV].

For $n \geq 3$, the following sufficient conditions for the existence and uniqueness of a minimal solution that tends to zero at infinity in some integral sense has been established in $[\mathrm{EK}]$ :

$$
\int_{\mathbb{R}^{n}} \frac{\rho(x) u_{0}(x)}{|x|^{n-2}} d x<\infty, \quad u_{0} \in L_{\mathrm{loc}}^{\infty}\left(\mathbb{R}^{n}\right) .
$$

In particular, this means that bounded solutions are not unique because the constant is not the minimal solution for constant initial data if $\int \rho(x)|x|^{2-n} d x<\infty$. The condition is met for densities $\rho$ as in our paper if $\alpha>2$. For $\alpha>n$, nonuniqueness follows at once from the energy loss (see Remark 6.4). 
The one-dimensional counterexample of Subsection 6.1 has been constructed for simplicity with a weight $\rho$ that is piecewise constant. The calculation with a power function seems more interesting, but it turned out too lengthy in our version.

There are many possible extensions of these results under different variations of the assumptions on the data. Thus, we can pose the (elliptic and parabolic) problems in a bounded domain with a weight $\rho$ that either blows up or degenerates at the boundary. In the latter case, it seems that certain growth conditions have to be imposed for our technique to work. The case of boundary blow-up is for instance used in proving the weak local smoothing effect for the planar logarithmic diffusion equation in [V6].

Acknowledgements. Both authors partially supported by Spanish Project BMF2002-04572-C02-02 and ESF Programme "Global and geometric aspects of nonlinear partial differential equations". We thank the referee for several helpful suggestions.

\section{References}

[AP] Aronson, D. G., Peletier, L. A.: Large time behaviour of solutions of the porous medium equation in bounded domains. J. Differential Equations 39, 378-412 (1981) Zbl 0475.35059 MR 0612594

[Ba1] Bandle, C.: On symmetrizations in parabolic equations. J. Anal. Math. 30, 98-112 (1976) Zbl 0331.35036 MR 0442477

[Ba2] Bandle, C.: Isoperimetric Inequalities and Applications. Pitman Adv. Publ. Program, Boston (1980) Zbl 0436.35063 MR 0572958

[BS] Bennett, C., Sharpley, R.: Interpolation of Operators. Pure Appl. Math. 129, Academic Press, Boston, MA, 1988 Zbl 0647.46057| MR 0928802

[BBMP] Betta, M. F., Brock, F., Mercaldo, A., Posteraro, M. R.: A comparison result related to Gauss measure. C. R. Math. Acad. Sci. Paris 334, 451-456 (2002) Zbl 1004.35044 MR 1890632

[BG] Boccardo, L., Gallouët, Th.: Nonlinear elliptic and parabolic equations involving measure data. J. Funct. Anal. 87, 149-169 (1989) Zbl $0707.35060 \mid$ MR 1025884

[BrK] Brezis, H., Kamin, S.: Sublinear elliptic equations in $\mathbb{R}^{n}$. Manuscripta Math. 74, 87-106 (1992) Zbl 0761.35027|MR 1141779

[BrS] Brezis, H., Strauss, W. A.: Semi-linear second order elliptic equations in $L^{1}$. J. Math. Soc. Japan 25, 565-590 (1973) Zbl 0278.35041 MR 0336050

[CL] Crandall, M. G., Liggett, T. M.: Generation of semi-groups of nonlinear transformations on general Banach spaces. Amer. J. Math. 93, 265-298 (1971) Zbl 0226.47038 MR 0287357

[CRZ] Crowe, J. A., Rosenbloom, P. C., Zweibel, J. A.: Rearrangements of functions. J. Funct. Anal. 66, 432-438 (1986) Zbl 0612.46027 MR 0839110

[E] Eidus, D.: The Cauchy problem for the non-linear filtration equation in an inhomogeneous medium. J. Differential Equtions 84, 309-318 (1990) Zbl 0707.35074 MR 1047572

[EK] Eidus, D., Kamin, S.: The filtration equation in a class of functions decreasing at infinity. Proc. Amer. Math. Soc. 120, 825-830 (1994) Zbl $0791.35065 \mid$ MR 1169025

[GM] Gallouët, Th., Morel, J.-M.: On some semilinear problems in $L^{1}$. Boll. Un. Mat. Ital. A (6) 4, 123-131 (1985) Zbl 0585.35033 MR 0781805 
[GHP] Guedda, M., Hilhorst, D., Peletier, M. A.: Disappearing interfaces in nonlinear diffusion. Adv. Math. Sci. Appl. 7, 695-710 (1997) Zbl 0891.35071 MR 1476273

[HLP] Hardy, G. M., Littlewood, J. E., Pólya, G.: Inequalities. Cambridge Univ. Press (1934) Zbl 0010.10703

[KK] Kamin, S., Kersner, R.: Disappearance of interfaces in finite time. Meccanica 28, 117120 (1993) Zbl 0786.76088

[KR1] Kamin, S., Rosenau, P.: Propagation of thermal waves in an inhomogeneous medium. Comm. Pure Appl. Math. 34, 831-852 (1981) Zbl 0458.35042 MR 0634287

[KR2] Kamin, S., Rosenau, P.: Nonlinear diffusion in a finite mass medium. Comm. Pure Appl. Math. 35, 113-127 (1982) Zbl 0469.35060 MR 0637497

[Kw] Kawohl, B.: Rearrangements and Convexity of Level Sets in PDE. Lecture Notes in Math. 1150, Springer, Berlin (1895) Zbl 0593.35002 MR 0810619

[LL] Lieb, E. H., Loss, M.: Analysis. 2nd ed., Grad. Stud. in Math. 14, Amer. Math. Soc., Providence, RI (2001) Zbl 0966.26002 MR 1817225

[RV] Reyes, G., Vázquez, J. L.: The Cauchy problem for the inhomogeneous Porous Medium Equation. In: Networks and Heterogeneous Media, to appear. Available at http://aimsciences.org/journals/NHM/index.htm

[SVS] Schulz, F., Vera de Serio, V.: Symmetrization with respect to a measure. Trans. Amer. Math. Soc. 337, 195-210 (1993) Zbl 0844.49025 MR 1088477

[T1] Talenti, G.: Elliptic equations and rearrangements. Ann. Scuola Norm. Sup. Pisa (4) 3, 697-718 (1976) Zbl 0341.35031 MR 0601601

[T2] Talenti, G.: Nonlinear elliptic equations, rearrangements of functions and Orlicz spaces. Ann. Mat. Pura Appl. 120, 159-184 (1979) Zbl 0419.35041 MR 0551065

[T3] Talenti, G.: Inequalities in rearrangement invariant function spaces. In: Nonlinear Analysis, Function Spaces and Applications, Vol. 5, M. Krbec et al. (eds.), Prometheus, Prague, 177-230 (1994) Zbl 0872.46020 MR 1322313

[T4] Talenti, G.: A weighted version of a rearrangement inequality. Ann. Univ. Ferrara Sez. VII (N.S.) 43, 121-133 (1997) Zbl 0936.26007 MR 1686750

[Tr] Trombetti, G.: Metodi di simmetrizzazione nelle equazioni alle derivate parziali. Boll. Un. Mat. Ital. Sez. B Artic. Ric. Mat. (8) 3, 601-634 (2000) Zbl 0963.35006 MR 1801615

[V1] Vázquez, J. L.: Symétrisation pour $u_{t}=\Delta \varphi(u)$ et applications. C. R. Acad. Sci. Paris 295, 71-74 (1982) Zbl 0501.35015 MR 0676365

[V2] Vázquez, J. L.: Symmetrization in nonlinear parabolic equations. Portugal. Math. 41, 339-346 (1982) Zbl 0524.35061 MR 0766862

[V3] Vázquez, J. L.: The Dirichlet problem for the porous medium equation in bounded domains. Asymptotic behavior. Monatsh. Math. 142, 81-111 (2004) Zbl 1055.35024 MR 2065023

[V4] Vázquez, J. L.: Symmetrization and mass comparison for degenerate nonlinear parabolic and related elliptic equations. Adv. Nonlinear Stud. 5, 87-131 (2005) Zbl pre02162932 MR 2117623

[V5] Vázquez, J. L.: Smoothing and Decay Estimates for Nonlinear Parabolic Equations of Porous Medium Type. Oxford Univ. Press, to appear

[V6] Vázquez, J. L.: Evolution of point masses by planar logarithmic diffusion. Finite-time blow-down. Preprint UAM (2005)

[W] Weinberger, H.: Symmetrization in uniformly elliptic problems. In: Studies in Mathematical Analysis and Related Topics, Stanford Univ. Press, 424-428 (1962) Zbl 0123.07202 MR 0145191 